\title{
Circadian orchestration of gene expression in cyanobacteria
}

\author{
Yi Liu, ${ }^{1}$ Nicholas F. Tsinoremas, ${ }^{2}$ Carl Hirschie Johnson, ${ }^{1}$ Nadya V. Lebedeva, ${ }^{2}$ Susan S. Golden, ${ }^{2}$ \\ Masahiro Ishiura, ${ }^{3,4}$ and Takao Kondo ${ }^{3-5}$ \\ ${ }^{1}$ Department of Biology, Vanderbilt University, Nashville, Tennessee 37235 USA; ${ }^{2}$ Department of Biology, Texas A\&M \\ University, College Station, Texas 77843 USA; ${ }^{3}$ National Institute for Basic Biology, Okazaki 444, Japan
}

We wanted to identify genes that are controlled by the circadian clock in the prokaryotic cyanobacterium Synechococcus sp. strain PCC 7942. To use luciferase as a reporter to monitor gene expression, bacterial luciferase genes $(\operatorname{lux} A B)$ were inserted randomly into the Synechococcus genome by conjugation with Escherichia coli and subsequent homologous recombination. The resulting transformed clones were then screened for bioluminescence using a newly developed cooled-CCD camera system. We screened $\sim 30,000$ transformed Synechococcus colonies and recovered $\sim 800$ clones whose bioluminescence was bright enough to be easily monitored by the screening apparatus. Unexpectedly, the bioluminescence expression patterns of almost all of these $\mathbf{8 0 0}$ colonies clearly manifested circadian rhythmicity. These rhythms exhibited a range of waveforms and amplitudes, and they also showed a variety of phase relationships. We also found bioluminescence rhythms expressed by cyanobacterial colonies in which the luciferase gene set was coupled to the promoters of several known genes. Together, these results indicate that control of gene expression by circadian clocks may be more widespread than expected thus far. Moreover, our results show that screening organisms in which promoterless luciferase genes have been inserted randomly throughout the genome by homologous recombination provides an extremely sensitive method to explore differential gene expression.

[Key Words: Bioluminescence; circadian rhythms; cyanobacteria; luciferase; luxAB genes; Synechococcus]

Received February 16, 1995; revised version accepted April 28, 1995.

Circadian (daily) rhythms regulate an enormous variety of biological phenomena, including behavior, metabolism, and gene expression (Bünning 1973; Kloppstech 1985; Dunlap 1993; Jacobshagen and Johnson 1994). These rhythms allow organisms to efficiently adapt to the day/night alterations of environmental illumination and temperature. As in development, where cell typespecific changes in gene expression allow different tissues to optimize their function, the circadian system programs a daily cycle of gene expression within each cell to optimize its function in a rhythmic environment. In the case of Neurospora, the comparison between developmental and circadian processes is not merely an analogy; the circadian clockwork controls the timing of a morphological differentiation, namely, the formation of aerial hyphae and conidiospores (Feldman and Dunlap 1983).

Another example of circadian programming of gene expression is the temporal separation of nitrogen fixation from photosynthesis in some cyanobacteria (Mitsui et al. 1986). These processes are mutually exclusive because the nitrogen-fixing enzyme nitrogenase is inhib-

${ }^{4}$ Present address: Department of Biology, Faculty of Science, Nagoya University, Furocho, Chikusa, Nagoya 464-01 Japan.

${ }^{5}$ Corresponding author. ited by the oxygen produced by photosynthesis. Nitrogen-fixing photosynthetic organisms have solved this dilemma in different ways. One solution is the spatial differentiation of specialized nonphotosynthetic cells, called heterocysts, which perform the nitrogen-fixing duties (Tandeau de Marsac and Houmard 1993). The temporal programming strategy, on the other hand, exploits the natural environment's support of photosynthesis only in the daytime. The circadian clock of cyanobacteria that use this latter strategy balances the expression of photosynthesis versus nitrogen fixation genes so that photosynthesis occurs during the day to take advantage of the sunlight, and nitrogen fixation moonlights during the dark period when oxygen levels within the cells are low (Grobelaar et al. 1986; Mitsui et al. 1986; Schneegurt et al. 1994). This temporal programming is accomplished by rhythmic gene expression, because it has been shown that the abundance of nitrogenase mRNA is higher during the night phase in these cyanobacteria (Huang and Chow 1990).

We are interested in how the circadian clock controls gene expression patterns and in ascertaining how widespread this temporal control is. Additionally, we would like to know whether the circadian pacemaker can turn genes on and off during a variety of phases. Other organisms are known to express rhythms of mRNA abundance 
(Kloppstech 1985; Kay and Millar 1992; Jacobshagen and Johnson 1994). In some organisms, subtractive hybridization of mRNAs expressed at different circadian phases has been used to discover previously uncharacterized clock-controlled genes (ccgs). For example, 12 ccgs have been discovered in Neurospora by subtractive hybridization, 2 of which have been reported (Loros et al. 1989; J. Loros, pers. comm.). Both of these ccgs appear to be regulated by the circadian clock via transcriptional control (Loros and Dunlap 1991), and one has been identified as a gene involved in the differentiation of conidiospores (Bell-Pederson et al. 1992). In higher plants, subtractive hybridization has revealed two other rhythmically expressed genes, both of which encode RNA-binding proteins (Heintzen et al. 1994).

Bioluminescence emitted by a luciferase reporter can be measured with exquisite sensitivity, providing an in vivo alternative to subtractive hybridization for estimating the extent of circadian control over gene expression. We have developed a method for random insertion of a luciferase reporter gene throughout the genome of the cyanobacterium Synechococcus sp. strain PCC 7942 with subsequent screening by a cooled-charge-coupled device (cooled-CCD) camera apparatus. Using the Vibrio harveyi luciferase genes (luxAB) as a reporter for the promoter activity of the $p s b A I$ gene, we have already shown that this cyanobacterium expresses circadian rhythms of gene expression (Kondo et al. 1993; Kondo and Ishiura 1994). The approach described herein allowed us to identify colonies of cyanobacteria in which the luciferase reporter had inserted into uncharacterized regions of the genome that induced expression of the luciferase and, subsequently, to ascertain whether the nearby promoter/ enhancer regions were regulated by the circadian clockwork.

Bioluminescence patterns from clones that carried random luciferase gene insertions showed that circadian control over gene expression in this cyanobacterium is more extensive than we had imagined. More than 800 clones expressed clear rhythms of bioluminescence, and the phasing of their rhythms showed a wide range of phase relationships. That the circadian clock regulates many genes in this organism was also supported by experiments in which the luciferase genes were coupled to the promoters of known Synechococcus genes. Therefore, the circadian system in this cyanobacterium exerts widespread control over gene expression with a wide variety of temporal phasing patterns.

\section{Results}

\section{Bioluminescence of colonies resulting from random insertion of luxAB}

We created a library of Synechococcus genomic DNA fragments fused to a promoterless lux $A B$ gene set that could be transferred to cyanobacteria by conjugation with Escherichia coli. This library was calculated to cover $99.9 \%$ of the Synechococcus genome (see Materials and methods). These sequences recombined into
Synechococcus by homologous recombination so that the region of DNA surrounding the insertion site is duplicated (Tsinoremas et al. 1994). Therefore, the insertion event should not disrupt the adjacent gene in most cases. Figure 1B shows one petri dish with colonies resulting from the conjugation procedure. Figure $1 \mathrm{~A}$ is the same plate whose bioluminescence image in complete darkness has been captured with the cooled-CCD camera. The distribution of the bioluminescent colonies on the dish looks like a galaxy visualized through a powerful telescope. Approximately $10 \%$ of the clones resulting from conjugation expressed bioluminescence at a level that was detectable with our cooled-CCD camera apparatus (Fig. 1, cf. A and B).

Figure $1 \mathrm{~A}$ also illustrates dramatically that the bioluminescence of some colonies is very bright, whereas that of other colonies is dim. This heterogeneity is not attributable to differences in colony size (Fig. 1B). Figure 2 shows the bioluminescence of 908 colonies that were recognized by our computerized colony recognition routine (Materials and methods). Most of the bioluminescence in the colonies is dim, with an approximately ex-

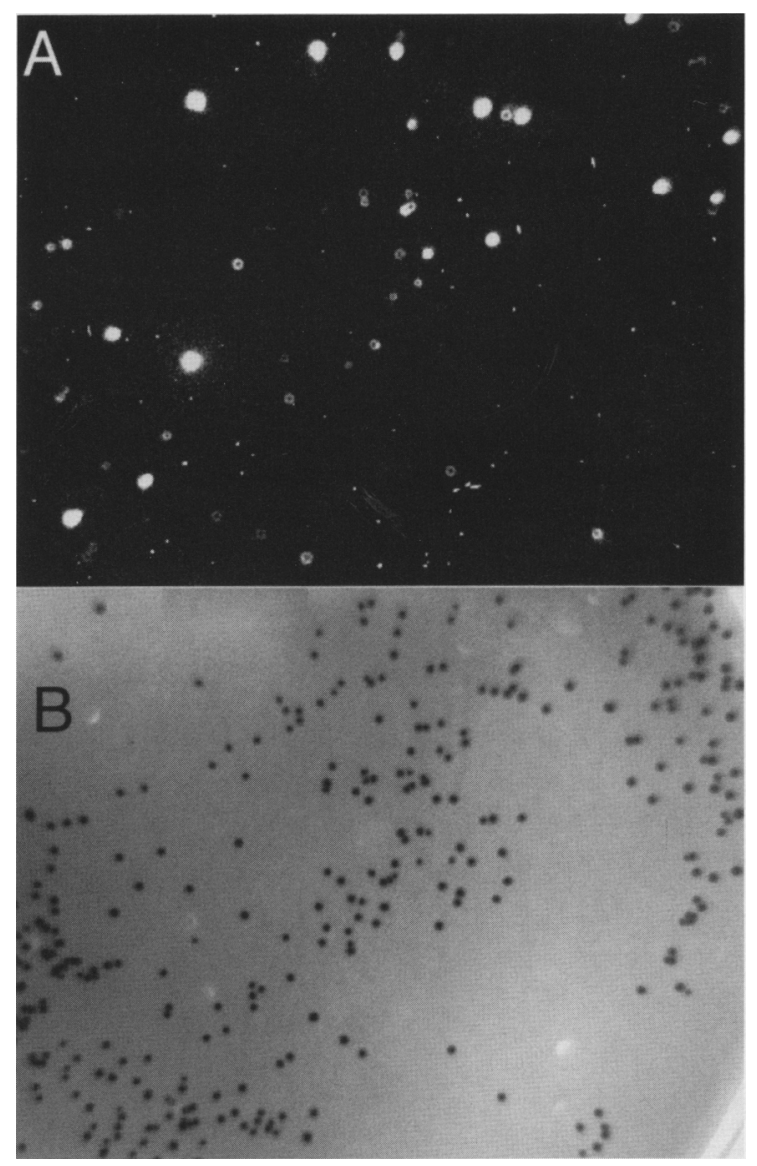

Figure 1. Bioluminescence of cyanobacterial clones resulting from conjugation. $(A)$ A plate viewed in complete darkness with the cooled-CCD camera. Bright spots indicate the locations of bioluminescent colonies. $(B)$ The same plate viewed under external illumination to show the location of every colony that has been transformed to a kanamycin-resistant phenotype. 


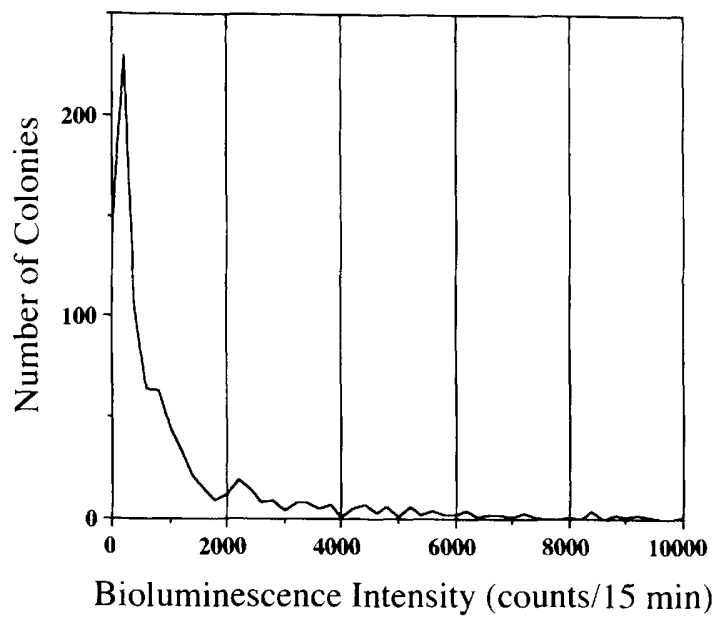

Figure 2. Histogram of the bioluminescence intensity distribution of primary colonies after conjugation. In this experiment, 9600 kanamycin-resistant colonies were screened for bioluminescence, and 908 colonies were found to have bioluminescence that was detectable by the cooled-CCD camera in a 15-min exposure. The intensity of bioluminescence of each of these 908 colonies ( $1 \mathrm{~mm}$ diam.) is plotted here. The abscissa is the bioluminescence intensity in counts captured by the cooled-CCD camera after a 15 -min exposure; the ordinate is the number of colonies expressing each intensity lorganized into bins of 200 counts).

ponential decline in the number of colonies exhibiting increasingly brighter bioluminescence. This distribution of intensities suggests that the lux $A B$ reporter gene set has randomly inserted into many areas throughout the genome near promoter regions, some of which are strongly active (the bright colonies) and others that are relatively weak (the dim colonies). Of $\sim 30,000$ colonies initially screened by the cooled-CCD apparatus, the time course of the bioluminescence of $\sim 800$ bright colonies and $40 \mathrm{dim}$ colonies was analyzed in greater detail.

\section{Bioluminescence profiles of bright clones}

Almost every "bright" colony expressed a clear circadian rhythm of bioluminescence in constant light (LL), although the waveforms of those rhythms were quite variable among different colonies. We classified the waveforms into five separate categories, which are illustrated in Figures 3 and 4. Classes 1, 2, and 3 exhibit approximately a symmetrical sine curve. The distinction between these three classes is based on their average phase: the trough of class 1 rhythms is at approximately circadian time 21-24 (CT 21-24), those of class 2 at approximately CT 11-14, and those of class 3 at approximately CT 17-20 (Figs. 3 and 4). The rhythm of our previous psbAI::luxAB construct-named AMC149 (Kondo et al. 1993, 1994) — exhibits a phase relationship that falls into class 1 . The waveforms of class 4 rhythms were significantly asymmetric, exhibiting a "sawtooth" pattern (Fig. 3 ). Finally, we grouped the remaining heterogeneous waveforms into class 5 . Two common waveforms in class 5 are those that are significantly bimodal and those that have "step-like" patterns (Fig. 3).

Figure 4 summarizes the phase relationships of the rhythms in the various classes. Taken together, those categories expressing symmetrical sine curve waveforms (classes 1-3) show that gene expression in Synechococcus can display a variety of phases, with preferences for troughs occurring at about CT 11-14 (class 2), CT 17-20 (class 3), and CT 21-24 (class 1). Sawtooth waveforms (class 4 , the largest class) primarily have their troughs at about CT 19-23. Class 5 waveforms bracket a range of phases from CT 18-3, but it should be remembered that phase determination for some class 5 rhythms lespecially the step-like patterns) is not as accurate as for the other waveforms.

The bottom of Figure 4 summarizes all of the phase data for the bright colonies (total $=789$ colonies). Clearly, rhythms can be expressed in a variety of phase relationships, with a strong preference for troughs at the end of the subjective night (CT 18-2) and a weaker preference for troughs in the early subjective night (about CT 10-15). These data demonstrate extensive temporal programming of gene expression in Synechococcus.

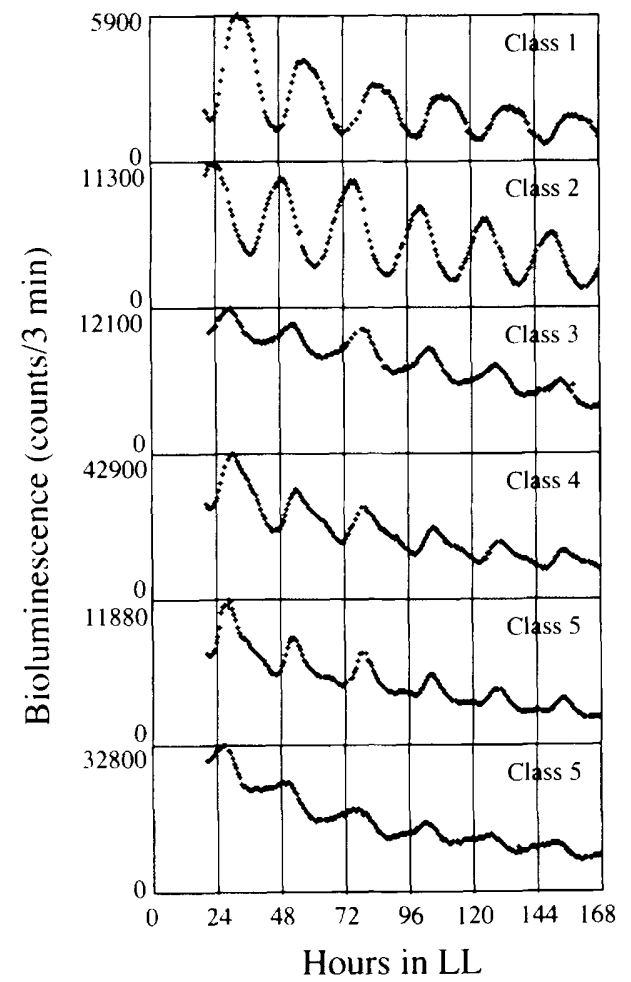

Figure 3. Representative traces of various classes of rhythmic waveforms expressed by bright colonies in LL. In comparison with Fig. 2, bright colonies are those expressing a level of bioluminescence of 2000 counts or more. In this experiment, the bright colonies were transferred from the primary plates and analyzed in a time-course experiment with the cooled-CCD apparatus. Bioluminescence intensity of a colony ( $3 \mathrm{~mm}$ diam.) is shown on the ordinate in counts of the cooled-CCD camera after a 3-min exposure. The distinctions between the five classes are explained in the text. 


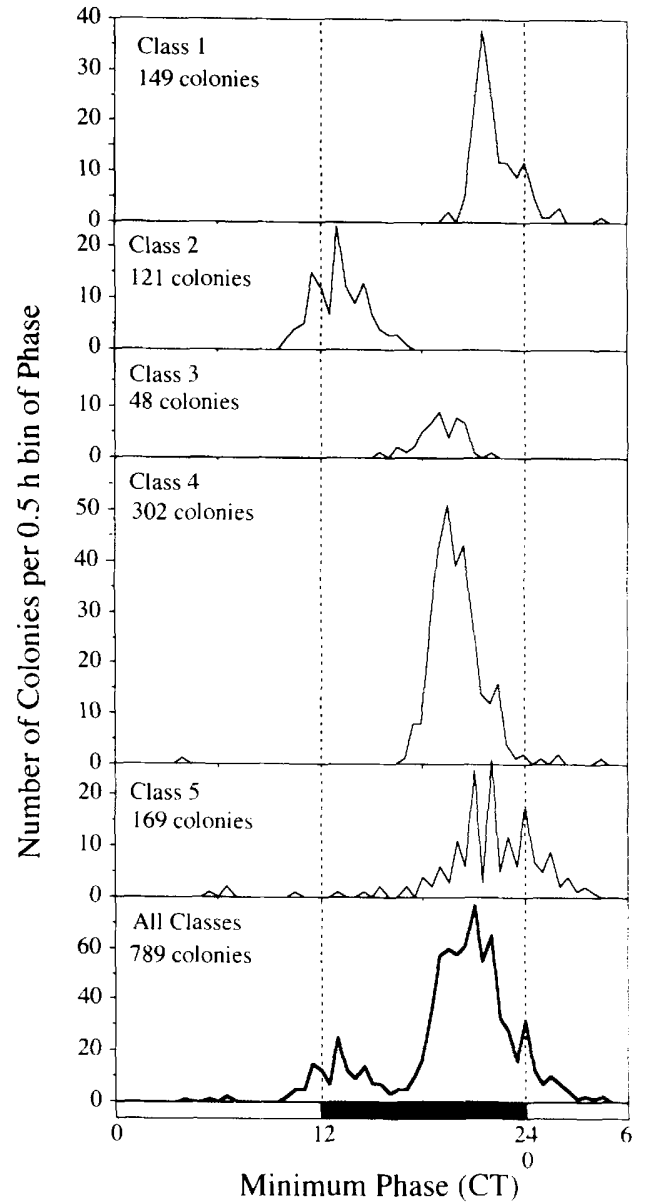

Figure 4. Distribution of phases for bioluminescence rhythms expressed by bright colonies in LL. The data are organized such that each panel depicts the phase distribution of one of the various classes of waveforms illustrated in Fig. 3. (Bottom panel) The composite of all the classes. The number of colonies in each class is indicated in each panel. (Abscissa) Phases of troughs of bioluminescence rhythms in CT, where CT 0 is the subjective dawn in LL, and CT 12 is the subjective dusk in LL (the white bar on the abscissa is the subjective day; the shaded bar is the subjective night). (Ordinate) Number of colonies (in bins of $0.5 \mathrm{hr}$ of phase) whose bioluminescence trough is at the indicated phase.

\section{Bioluminescence profiles of dim clones}

The temporal pattern of bioluminescence was also checked for some colonies whose light emission was too low to be tracked efficiently in a time-course experiment with our cooled-CCD apparatus. We transferred $40 \mathrm{dim}$ colonies to a photon-counting photomultiplier apparatus that was much more sensitive to low levels of bioluminescence (Materials and methods) and found that they also glowed rhythmically (Fig. 5). Although our analysis of these dim clones was much less extensive than for the bright clones, it appears that the phase of most of the dim clones is like that of the class 1 bright colonies (Fig. 5, A-Cl. However, one clone showed a rhythm whose phase appears to be class 2 (Fig. 5E), and two clones had a waveform similar to class 4 (one of which is depicted in Fig. 5D).
Confirmation of multiple-site insertions of the luxAB gene set

To confirm that the $\operatorname{lux} A B$ gene set had inserted into many sites throughout the Synechococcus genome, DNA was extracted from five class 1 clones. The DNA was digested with BamHI and EcoRI, and a Southern blot experiment was performed using lux DNA as the probe (Fig. 6). The hybridization patterns were different among the transconjugant strains, indicating that the $\operatorname{lux} A B$ genes have been introduced into different regions of the genome even within clones of the same phase-relationship category. As the hybridization pattern of each clone is distinct, these data confirm the conclusion that our strategy resulted in the integration of the lux $A B$ gene set into many sites throughout the Synechococcus genome. Sequence analysis indicated that the insertion site was also different for two randomly chosen class 2 clones (data not shown). However, Southern analysis of a sampling of class 2 clones indicated that one insertion site is

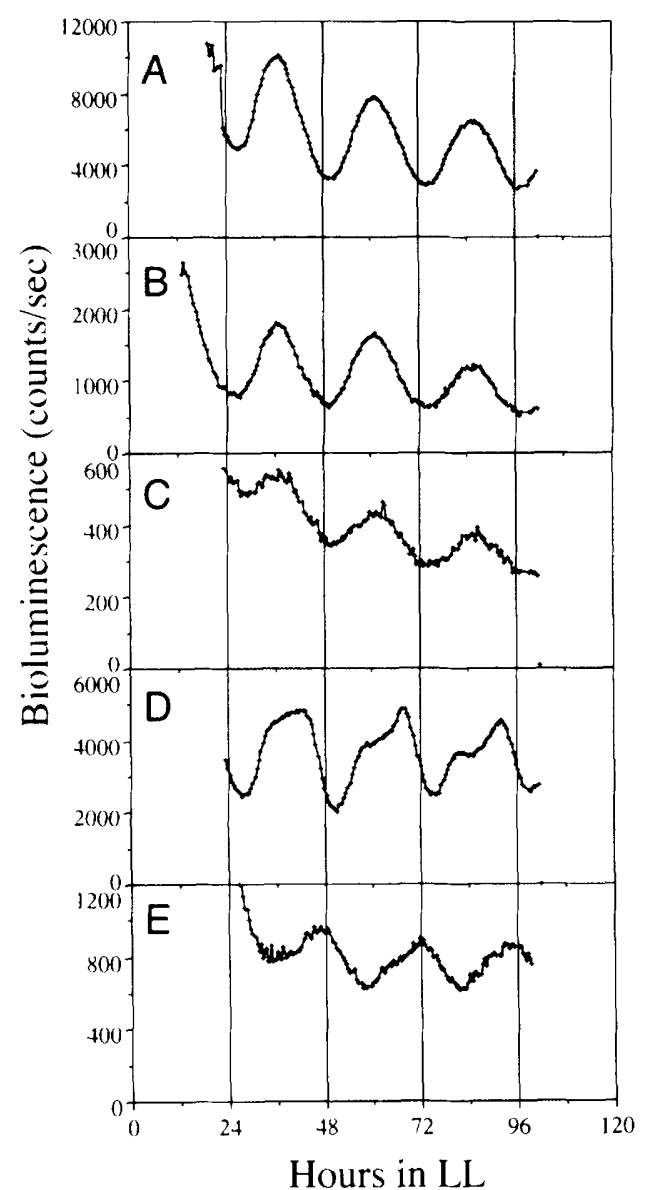

Figure 5. Representative traces of dim colonies. In comparison with Fig. 2, dim colonies are those expressing a level of bioluminescence of $<2000$ counts on the abscissa in Fig. 2. Bioluminescence was measured as described in Materials and methods. Units of bioluminescence on the ordinate are counts/sec of the photon counter and correspond to $1 / 30$ to $1 / 100$ of units on the ordinate in Fig. 3. 


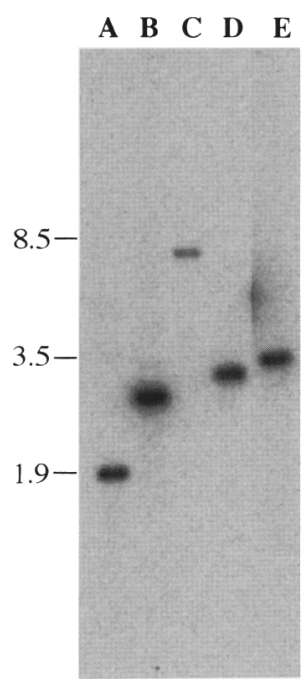

Figure 6. Southern blot analysis to confirm multiple-site insertions of the luxAB genes. Genomic DNA from five indepen dent clones belonging to class 1 was extracted and digested with $B a m H I$ plus EcoRI. Southern blot analysis was performed as described in Materials and methods. These hybridization signals represent DNA from an EcoRI site near the start codon of $\operatorname{lux} B$ to the nearest $E c o$ RI or BamHI site upstream of $\operatorname{lux} A$ in the Synechococcus genome. Lane $A$ is DNA from our previous psbAI::luxAB strain (AMC149; Kondo et al. 1993), Lanes $B, C$, $D$, and $E$ are DNAs from four class 1 recombinant strains. Molecular sizes (in $\mathrm{kb}$ ) are indicated for the migration of three relevant bands of a standard that was generated by digestion of bacteriophage $\lambda$ DNA with BstEII

over-represented, and thus, class 2 clones may be rarer than Figure 4 suggests.

\section{Confirmation of rhythmic gene expression by Northern analysis}

We demonstrated previously that one class 1 gene, psbAI, shows a rhythm of promoter activity and a rhythm of mRNA abundance (Kondo et al. 1993; Liu et al. 1995), and that $\operatorname{lux} A B$ functions as an excellent reporter of class 1 promoter activity in Synechococcus (Liu et al. 1995). To confirm that luxAB functions as a reliable reporter of transcription for genes of other expression classes, we measured the mRNA abundance of a class 2 gene that we have identified (Y. Liu, unpubl.). This gene is $\operatorname{pur} F$, which encodes the enzyme catalyzing the initial step of the de novo purine nucleotide biosynthesis pathway, amidophosphoribosyltransferase (Markaroff et al. 1983; Ebbole and Zalkin 1987). Although the purF message is of low abundance and appears to be unstable, its level oscillates in antiphase to that of the class 1 psbAI mRNA (Fig. 7). These data indicate that lux $A B$ accurately reports transcriptional activity of genes that are expressed at different phases of the circadian cycle.

\section{Rhythmicity of bioluminescence from lux $\mathrm{AB}$ fusions to known genes}

In addition to the random insertion strategy to identify clock-controlled genes, we also prepared fusions of a promoterless lux $A B$ gene set to several promoters of Synechococcus genes that had already been identified. We chose three promoters that we expected to express different patterns of expression over the circadian cycle. The first was psbAIII lencoding the Dl protein of Photosystem II), whose response to high light is different from that of the class 1-type $p s b A I$ gene that we have used extensively (Kondo et al. 1993, 1994) and whose promoter has been well characterized ( $\mathrm{Li}$ and Golden 1993). The second was $g \ln A$ (glutamine synthetase), which is not light regulated (Bustos et al. 1990), and the third was the promoter for the rRNA gene $(r r n A$; Kumano et al. 1986), which we expected to be constitutive.

As shown in Figure 8, constructs of all three genes exhibited class 1 phase and waveform under our screening conditions. These data are consistent with those of the random insertion library; when measured by a sufficiently sensitive method, every promoter that we have tested shows at least a low amplitude rhythm. Additionally, these promoters are from well-characterized genes, so the data in Figure 8 confirm that rhythmic bioluminescence patterns are not a bizarre artifact of the random insertion protocol. Together, these data support the idea of extensive circadian control over gene expression.
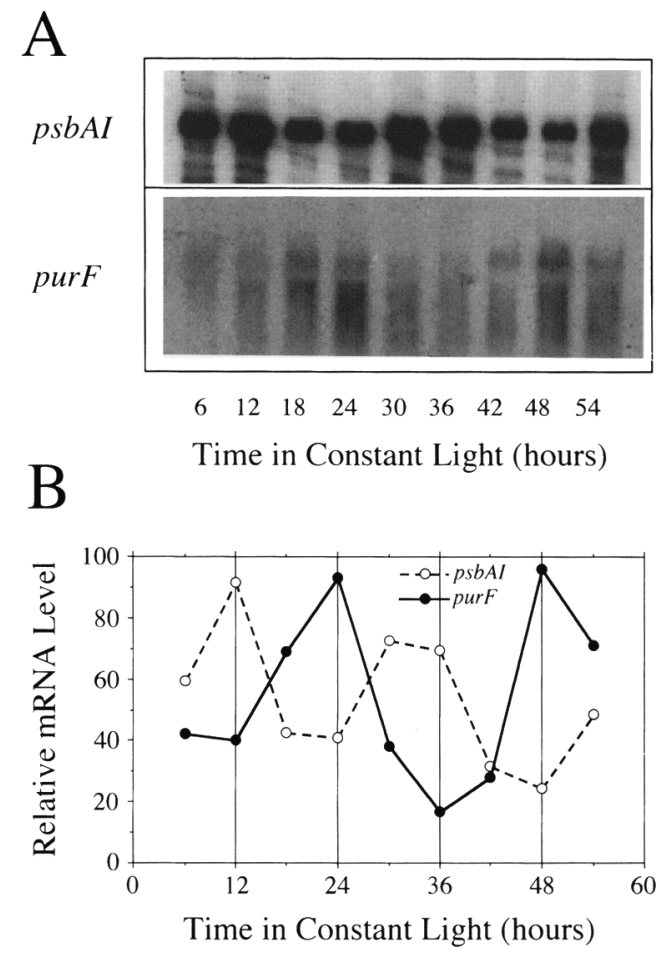

Figure 7. Rhythms in LL of mRNA abundance of a class 1 gene $(p s b A I)$ vs. a class 2 gene (purF) in wild-type Synechococcus. $|A|$ Northern blots of $p s b A I$ and purF mRNAs as a function of time in $L L$. (B) Densitometric data of the blots shown in $A$. 


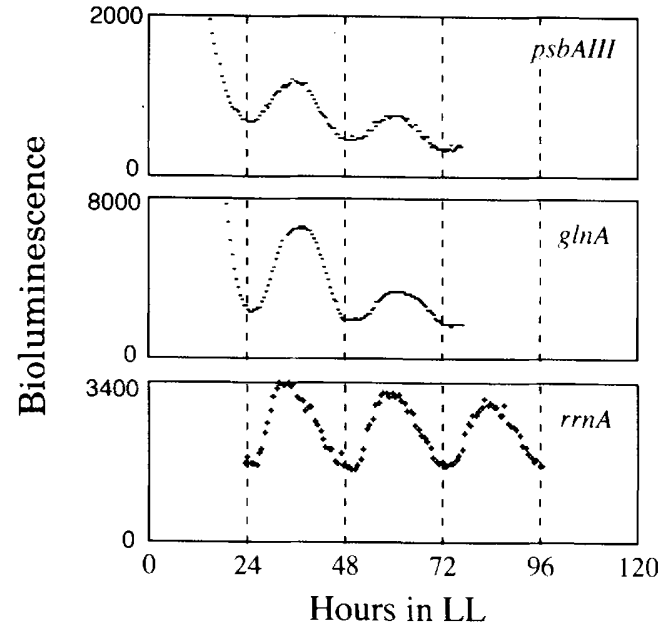

Figure 8. Traces of bioluminescence expressed from promoters of known genes. Bioluminescence was monitored from Synechococcus strains that carry luxAB gene fusions to the psbAIII, $g \ln A$, or $\operatorname{rrn} A$ promoters. The $p s b A I I I$ and $g \ln A$ reporter strains were measured as for the dim random library clones, whereas the $\operatorname{rn} A:: \operatorname{lux} A B$ strain was measured by the cooled-CCD apparatus used for the bright clones. Ordinates of psbAIII and $\operatorname{gln} A$ are the same as those in Fig. 5, and $\operatorname{rrnA}$ ordinates are the same as those in Fig. 3.

\section{Discussion}

\section{Extensive circadian modulation of gene expression}

This investigation is the first overview of promoter activity in a genome over the circadian time scale. This feat was accomplished by virtue of the high efficiency of our gene transfer protocol via conjugation and by continuous monitoring of thousands of individual colonies by the cooled-CCD camera apparatus. The most striking result is that almost all of the clones that expressed bioluminescence showed a clear circadian rhythm. In addition, on the basis of the bright clones alone, the rhythms showed a variety of phase relationships. The data from the dim clones, although not as extensive as those of the bright clones, suggest that if a sufficiently large number of dim colonies were analyzed, the distribution of waveforms and phases of the dim colonies' rhythms may be similar to that of the bright colonies. These facts plus the results of our Southern blots (see Fig. 6) show that most of the colonies that we have screened are the result of linking the lux $A B$ reporter construct to different promoters (see below).

In comparison with the results of searches in other organisms for clock-controlled genes, an unexpected result of our study is that almost every bioluminescent colony expressed a clear rhythmic pattern, and none was definitively arhythmic. Thus, circadian gene expression in Synechococcus is pervasive. The circadian clock in cyanobacteria does not merely control a specialized set of genes, but it dominates the entire metabolism of the cell.

Why do our results differ from those of clock researchers working with eukaryotic organisms? Of course, it is possible that circadian modulation of gene expression in the prokaryotic cyanobacteria is more extensive than in eukaryotes. On the other hand, perhaps when enough colonies (or individuals) can be screened by a method of high sensitivity in intact cells of other organisms, circadian clock control will be found to be widespread. Consistent with this idea is the observation that some genes in eukaryotic organisms are constitutively expressed under some conditions but rhythmically expressed under other conditions, for example, the cytochrome $c$ gene in Chlamydomonas (Jacobshagen and Johnson 1994). Another possible explanation is that our screen was for rhythmic promoter activity, not rhythmic mRNA abundance; perhaps clock control of transcription is widespread, but post-transcriptional effects sometimes mask rhythmic promoter activation. A support for this interpretation comes from experiments with one of the $c a b$ genes of Arabidopsis, which shows no rhythm of mRNA abundance, but transcriptional fusions of its promoter to a reporter gene indicate that the promoter is rhythmically activated by the circadian clock (Millar and Kay 1991).

Although it is clear that we have tagged many different genes in our screening, it is difficult to make an exact estimate of the number of genes that have been assayed. The library of Synechococcus DNA fragments generated by Sau3AI cleavage was calculated to cover $99.9 \%$ of the genome (Materials and methods). The conjugation method that we used is very efficient (Tsinoremas et al. 1994), and the genome of Synechococcus sp. PCC 7942 is small (2.7 Mb; M. Sugiura, pers. comm.), so the coverage of the genome theoretically should be excellent. Our Southern blot data suggest that many of the insertions of the $\operatorname{lux} A B$ gene set are unique, which supports the conclusion that most of the rhythmically bioluminescent colonies are the result of insertions into different genomic loci.

There are several reasons why our coverage of the genome is not likely to equal the theoretical $99.9 \%$, however. For example, some Synechococcus DNA segments could carry a coding sequence for a protein that interferes with $E$. coli metabolism, so that such segments might have been lost during propagation in $E$. coli. Also, insertion of the antibiotic-resistance sequence, a random fragment of Synechococcus DNA, and the luxAB gene set may disrupt gene expression in some locations in the Synechococcus genome. Nevertheless, our calculations plus (1) the heterogeneity of Southern blot patterns of both the E. coli library cells and the target Synechococcus clones (Fig. 6), and (2) the large range of bioluminescence patterns expressed by the bright colonies indicates that a large enough proportion of the genes in Synechococcus has been tagged to make a statistically valid statement about the extensive influence of the circadian clock over gene expression in this cyanobacterium.

\section{Phased gene expression}

Class 1 colonies express a rhythm whose phase is very close to that of our previously constructed psbAI::lux $A B$ 
reporter strain (named AMC149), in which the bioluminescence increases progressively during the subjective day and peaks near subjective dusk, with a corresponding trough near subjective dawn (Kondo et al. 1993). The phasing displayed by classes $1,3,4$, and 5 genes covers a broad but similar range /although assigning a definite phase to the variable waveforms of some of the class 5 rhythms is difficult). Therefore, up to $85 \%$ of the bright colonies oscillated with this phase relationship, as did most of the dim clones we tested. Because many clones oscillated with this approximate phasing, perhaps this range is a kind of "default" phase of rhythmic gene expression. If this phase is maintained in a light/dark cycle, then these genes would be expected to be most active in the daytime. Under environmental light/dark cycle conditions, the energy metabolism of these cells should be most active during the day in this photoautotrophic organism, so it is not surprising that many rhythmic genes should exhibit this phase relationship. Additional support for a default phase of gene expression is provided by studies in which we fused promoters with no additional cis-acting elements to the lux $A B$ gene set and observed circadian rhythms with class 1 patterns (data not shown).

Because the daily cycle of sunlight is crucially important to cyanobacteria, we would predict that some genes might be controlled by the circadian clock to anticipate dawn, thereby maximizing the harvesting of light energy. Class 2 clones (15\% of the total screened) apparently reflect genes of precisely this type: Their rhythms of bioluminescence peak in early subjective day (trough in early subjective night). The bioluminescence of class 3 rhythms peaks in the mid-subjective day (trough in mid- subjective night). Class 3 colonies numbered $6 \%$ of the total colonies screened. Our data support the hypothesis that efficient temporal organization in cyanobacteria can be achieved by programming some genes to be expressed at a variety of phases ranging from late subjective night throughout the subjective day under constant conditions.

An interesting result was that no or very few colonies expressed a rhythm with a minimum phase in the first three-quarters of the subjective day (CT 3-9), which would correspond to peak activity in the first three-quarters of the subjective night (CT 12-21). For an autotrophic photosynthetic organism, it is possibly significant that gene expression is relatively quiescent during the phase of the daily cycle in which light energy is un available. Because we screened a large number of clones, the absence of rhythms with this phase probably reflects a true "silent" phase and is not an artifact of having missed such colonies.

\section{Control of gene expression by the circadian clock}

Because we observed rhythms of gene expression that show a variety of phase relationships, some of these genes are likely to be directly regulated by the clock via cis-acting elements and trans-acting factors that coordinately regulate the expression of single genes or groups of genes (Fig. 9). In the simplest version of this scenario, there would be one (or a few) types of class 1-specific cis-acting elements turned on by a class 1 -specific transacting factor expressed by the clock during the day. A different set of class 2-specific cis-acting elements would be turned on by a class 2 -specific trans-acting factor that the circadian oscillator induces at night, and so forth.
Figure 9. Model for circadian control of gene expression in Synechococcus. Coding regions of genes are depicted as rectangles; adjacent promoter regions are depicted as boxes with arrowheads. Sine curves illustrate the phasing of various overt rhythms (far right) and rhythms of trans-acting factor/nonspecific factor activity (center). The circle (left) is the central circadian clockwork. Some ensembles of genes may be controlled by rhythmic activity of specific trans-acting factors that coordinately regulate groups of genes (top two diagrams|. These trans-acting factors could be activated at different specific phases (note the difference in phase between activity rhythms of trans-acting factors A and B|. Promoters responding to specific trans-acting factors may be regulated coordinately, e.g., open-box promoters respond to trans-acting factor $\mathrm{A}$, closed-box promoters respond to trans-acting factor B. Other genes, especially those in the default phase, may be regulated by global, but nonspecific factors that oscillate, as illustrated in the bottom diagram. In the latter case, promoter regions of the regulated genes may vary but still be rhythmically activated by nonspecific global factors.

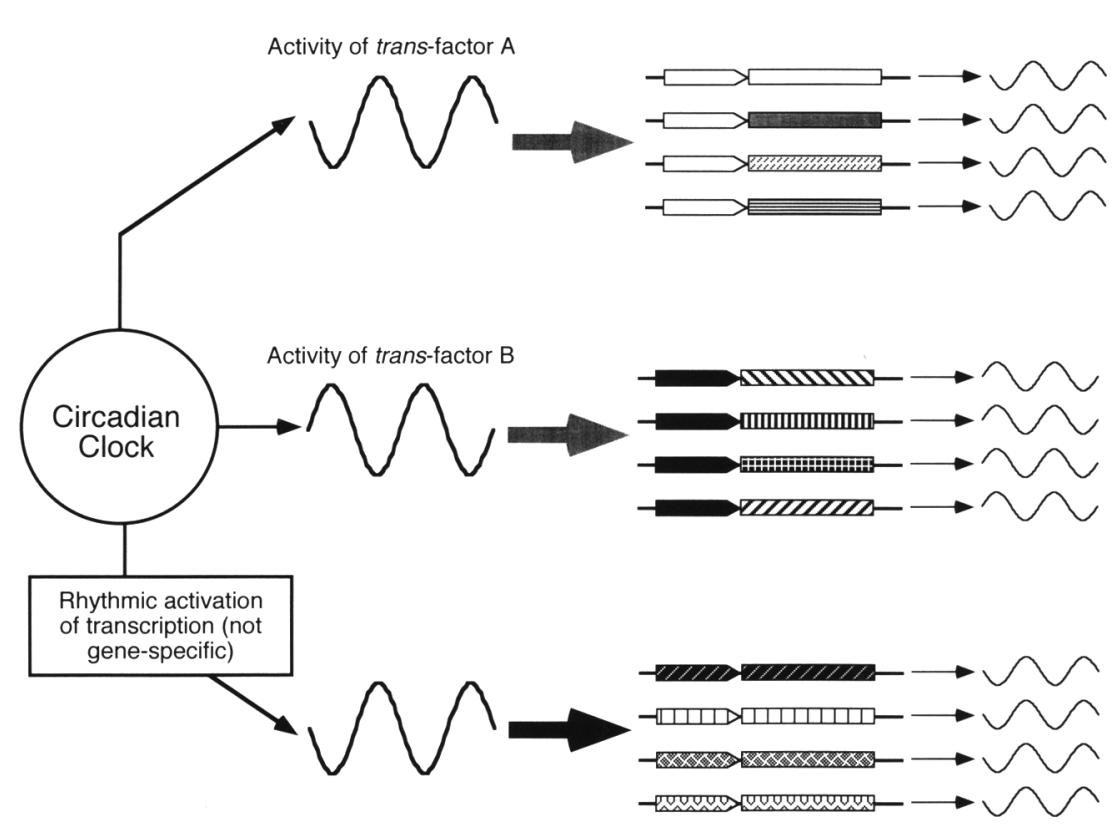


Because of the large number of genes that are apparently influenced by the clock in cyanobacteria, however, it seems unlikely that each of them is controlled by a specific regulatory factor. Some global factors could be involved as well. For example, because the energy status of these cells is dependent on photosynthesis, energy charge will certainly oscillate during the daily cycle, and this could influence the transcriptional rate of many genes, perhaps resulting in a waveform with the default phase as depicted in Figure 9. Another candidate for nonspecific control could be the circadian control of a global event, such as DNA replication. If the rate of DNA replication undergoes circadian modulation, then overall transcription rates could also be affected (Theisen et al. 1993|. Another global but nonspecific factor might be the status of the supercoiling of the chromosome; in Chlamydomonas, the supercoiling of the chloroplast's chromosome is influenced by light/dark conditions (Thompson and Mosig 1990). On the other hand, bioluminescence rhythms that are the result of such a nonspecific factor would not be expected to display a variety of phase relationships and waveforms. Therefore, the data are best explained by a model that incorporates both nonspecific circadian control and circadian regulation by specific trans-acting factors.

\section{Further analyses of rhythmic colonies in Synechococcus}

An important question about the organization of circadian systems is the elucidation of the mechanism by which the clock controls rhythmic gene expression. The different phasic classes of rhythmic gene expression revealed by our screening indicates that there are at least several independent regulatory signals issued by the clock. Presently, we do not know the identities of most of the genes that are linked to the clock.

Reporter constructs provide a powerful tool to answer this question. Because the insertion sites are tagged with the luxAB, kanamycin-resistance, and pBR322 sequences, the adjacent regions of Synechococcus DNA can be recovered in E. coli as a kanamycin-resistance plasmid and sequenced (Tsinoremas et al. 1994). This procedure can identify the relevant gene and promoter. As mentioned above (and see Fig. 7), we have identified one of the class 2 genes by this technique and found it to be the $\operatorname{pur} F$ gene encoding the rate-limiting enzyme of the purine nucleotide biosynthetic pathway, amidophosphoribosyltransferase (Markaroff et al. 1983; Ebbole and Zalkin 1987). We plan to carry out this rescue procedure for other representative clones of each class to better understand circadian orchestration of gene expression.

Random insertion of promoterless luciferase genes, followed by screening of bioluminescence patterns, is an elegant and sensitive method of screening for differential gene expression in general. This method is particularly applicable to studies of development and responses to environmental stress (Wolk et al. 1991). By using a sensitive cooled-CCD camera, both temporal and spatial patterns of gene expression can be detected.

\section{Materials and methods}

\section{Strain and growth conditions}

Wild-type Synechococcus sp. strain PCC 7942 was cultured in modified (Bustos and Golden 1991) BG-11 medium (Allen 1968) under constant illumination $\left[50-70 \mu \mathrm{E} / \mathrm{m}^{2}\right.$ per $\sec (\mathrm{E}$ is the energy of 1 mole of photons $\|$ at $30^{\circ} \mathrm{C}$. Agar plates $(1.5 \%)$ with modified BG-1l medium plus $5 \%$ Luria broth $(\mathrm{vol} / \mathrm{vol})$ were used for conjugation (Tsinoremas et al. 1994).

Construction of the DNA library and transfer to Synechococcus

A 2.8-kb SmaI fragment of plasmid pAM1040 (C.A. Strayer and S.S. Golden, unpubl., based on pLAV1; Chlumsky 1991), which carries the promoterless carboxy-terminal coding region of luxD and the intact $\operatorname{lux} A$ and $\operatorname{lu} \times B$ genes, was isolated and inserted into the EcoRV site of the mobilizable vector pAMI 153 (a modified pBR322 plasmid that encodes kanamycin resistance; Tsinoremas et al. 1994). The resulting plasmid, pAM1224, in which the luxAB and kanamycin-resistance genes have the same orientation, was linearized upstream of luxA by digestion with BgIII and dephosphorylated with calf intestinal phosphatase (Promega). Genomic DNA from Synechococcus sp. strain PCC 7942 was isolated as described by Bustos et al. (1990), partially digested with Sau3AI, and separated by electrophoresis on $1 \%$ agarose gels (Sambrook et al. 1989). Genomic DNA fragments of $1.5-4 \mathrm{~kb}$ were isolated from the gels and purified. Approximately $100 \mathrm{ng}$ each of the pooled DNA fragments and the BgIII-digested/dephosphorylated pAM1224 was mixed and treated with T4 DNA ligase. An aliquot of the ligation mixture was introduced into $E$. coli $\mathrm{DH} H \mathrm{~B}$ cells by electroporation (Cell Porator, BRL) and kanamycin-resistant E. coli colonies were selected on agar plates. DNA was prepared from $>50$ randomly chosen $E$. coli transformants. Digestion with EcoRI confirmed that $\sim 85-90 \%$ of the transformants contained plasmids with random restriction patterns (data not shown). These data suggest that the inserts were random samples of the Synechococcus genome.

The remainder of the ligation mixture was introduced into $E$. coli strain AM179, which contains the conjugation helper plasmid pRL528 and is chloramphenicol resistant (Elhai and Wolk 1988). More than 10,000 transformants resistant to kanamycin $(50 \mu \mathrm{g} / \mathrm{ml})$ and chloramphenicol $(170 \mu \mathrm{g} / \mathrm{ml})$ were collected by washing plates with $4 \mathrm{ml}$ of antibiotic-containing LB medium to form a chromosomal DNA: luxAB library. The library was stored in aliquots in the presence of $15 \%$ glycerol at $-70^{\circ} \mathrm{C}$ until needed for conjugation. The genome size of Synechococcus is $\sim 2.7 \mathrm{Mb}$, and the average size of the inserts was $\sim 2.7 \mathrm{~kb}$; statistically, therefore, this library should contain $>99.9 \%$ of the Synechococcus genome.

Transfer of this library into Synechococcus cells was achieved by triparental conjugal mating (Elhai and Wolk 1988; Tsinoremas et al. 1994). The cells were spread on agar plates containing BG- 11 plus $5 \% \mathrm{LB}(\mathrm{vol} / \mathrm{vol})$. After incubating overnight under LL $\left(\sim 60-70 \mu \mathrm{E} / \mathrm{m}^{2}\right.$ per sec) at $30^{\circ} \mathrm{C}$, the plates were underlaid with kanamycin $(10 \mu \mathrm{g} / \mathrm{ml}$, final concentration). After $\sim 2$ weeks, $>20,000$ kanamycin-resistant colonies were collected by washing the plates with BG-11 medium containing kanamycin. The cells were replated on BG-11 plates, allowed to develop kanamycin-resistant colonies, and screened for bioluminescence as described below. This replating procedure excluded transconjugants whose growth was slowed by the insertion. Because the average size of the insert is $\sim 2.7 \mathrm{~kb}$ and the insertion results in a duplication after a single homologous recombina- 
tion (Fig. 10), in most cases the endogenous genes should not be interrupted by the insertion of the DNA::lux $A B$ constructs.

\section{Screening method}

Transformed cells ( 2000 cells) were spread on 9-cm plates of BG-11 agar medium. After incubation in LL at $26^{\circ} \mathrm{C}$, the bioluminescence of plates was captured by a cooled-CCD detector (TE/CCD512BKS, Princeton Instruments) with an exposure of 15 or $20 \mathrm{~min}$. Selected bright bioluminescent colonies were transferred as single colonies to new plates with sterile toothpicks. After incubation for 4-5 days in LL and a 12-hr dark exposure to synchronize the circadian clocks in the various colonies, bioluminescence of colonies was monitored continuously by an automated monitoring system (Kondo et al. 1994) in LL for 4-5 days. The position of the trough of the rhythm was computed by a regression procedure similar to that used for the rhythm of Chlamydomonas (Kondo et al. 1991). The positions of the first troughs were estimated by extrapolation of times of troughs in LL and expressed in CT scale. CT 0 is the subjective dawn in $\mathrm{LL}$, and CT 12 is the subjective dusk in $\mathrm{LL}$.

Selected dim bioluminescent colonies were transferred to $1-\mathrm{cm}^{2}$ patches on new plates. After incubation for 4-5 days in LL and a 12-hr dark exposure, each patch was transferred to a $20-\mathrm{ml}$ scintillation vial. The bioluminescence from the vials was measured by an automated photomultiplier tube apparatus described previously (Kondo et al. 1993), except that the sensitivity was increased by routing the output to a photon counter. A low-noise photomultiplier (Hamamatsu R2693P) was used at $1000 \mathrm{~V}$, and this signal was processed by a photon-counting unit (Hamamatsu C3866) that removes thermal noise by discrimination of peak heights (signal at peak heights of $0.2 \mathrm{~V}$ or above). Signal pulses from the C3866 were detected by an electronic counter (Iwatsu SC-7201). Because of (1) the high gain of the R2693P photomultiplier tube, and (2) the discrimination between thermal noise and signal afforded by the photon counter, the effective sensitivity of light detection was 30-100 times greater than the previous analog system reported by Kondo et al. (1993).

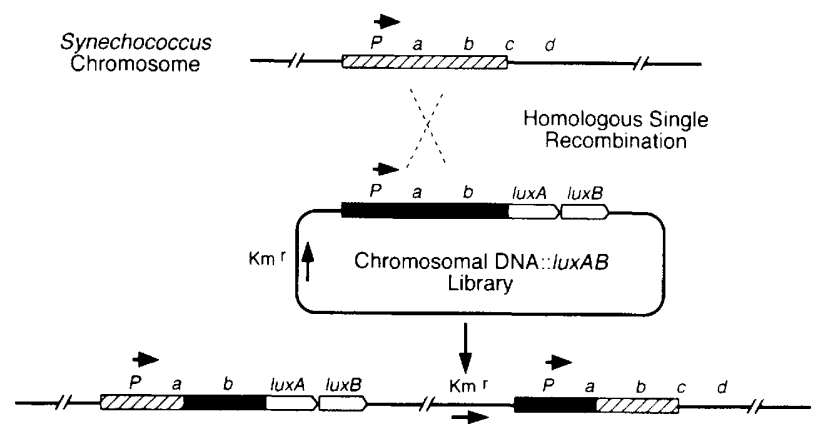

Figure 10. Integration of the random $\operatorname{lux} A B$ gene library into the Synechococcus chromosome. The chromosomal DNA::lux $A B$ library was introduced into Synechococcus by conjugation. After a single homologous recombination, the DNA inserts in the library are duplicated as shown and the lux $A B$ genes are inserted into the genome. The $1.5-$ to $4.0-\mathrm{kb}$ fragments of the Synechococcus chromosome are indicated by the solid bars. The hatched bar denotes the homologous fragment in the wild-type Synechococcus chromosome. $\left(\mathrm{Km}^{\mathrm{r}}\right)$ The kanamycin-resistance gene; $(P)$ The promoter of a hypothetical operon; $(a, b, c, d)$ different regions of that operon.
Southern blotting analysis of DNA from different Synechococcus clones

Minipreps of total DNA (Bustos et al. 1990) were prepared from randomly chosen bioluminescent clones from class 1. Each sample $(-250 \mathrm{ng})$ was digested with BamHI plus EcoRI and subjected to electrophoresis on $1 \%$ agarose gels. DNA was transferred onto nylon membranes (Hybond-N, Amersham; Sambrook et al. 1989). An EcoRI fragment of pAM1040 that includes all of $\operatorname{lux} A$ and $32 \mathrm{bp}$ of $\operatorname{lux} B$ was labeled with $\left[\alpha^{-32} \mathrm{P}\right] \mathrm{dCTP}$ by the random primer method (DECAprime DNA labeling kit from Ambion, Inc.) and used as a probe.

\section{Northern blotting analysis}

For the experiment depicted in Figure 7, wild-type cyanobacteria were grown at $30^{\circ} \mathrm{C}$ in $L L$ to an approximate cell density of $O D_{750}=0.5$ and given a $12 \mathrm{hr}$ exposure to darkness to synchronize the cells. Beginning $6 \mathrm{hr}$ after the reinitiation of LL conditions, $40-\mathrm{ml}$ samples were collected every $6 \mathrm{hr}$ for $54 \mathrm{hr}$ by centrifugation, frozen quickly in liquid nitrogen, and stored at $-70^{\circ} \mathrm{C}$. After all samples had been collected, Northern blots of total RNA were prepared as described previously (Liu et al. 1995). The membrane was first hybridized with a radioactive probe for psbAI mRNA (Liu et al. 1995), and the blot was then stripped of $p s b A I$ probe and hybridized with a radioactive probe for purF mRNA. The purF probe was an antisense RNA that specifically recognizes the coding region of the purF gene, and it was labeled with $\left[\alpha-{ }^{32} \mathrm{P}\right] \mathrm{UTP}$ by using an in vitro transcription kit (Ambion, Inc.). A computerized densitometer (Technology Resources $\mathrm{MC1000}$ ) was used to quantify the autoradiographs of the Northern blots.

\section{Fusion of luxAB to promoters of known genes}

A promoter assay vector (pAM1293) was constructed in which promoter-bearing fragments can be inserted upstream of the promoterless luxAB gene set and targeted to a neutral site of the Synechococcus genome (Bustos and Golden 1992). A 2.8-kb SmaI-PvulI fragment containing promoterless $\operatorname{lux} A B$ (from pAM1040, see above) was inserted into the SmaI site of pAM854 (Bustos and Golden 1991). Promoter fragments were inserted into a unique $S m a I$ site upstream of the luxA gene. The $p s b A I I I$ promoter/enhancer fragment $(-38$ to +39 ; Li et al. 1993) was generated by PCR, as was a 195-bp fragment containing the $r \mathrm{rn} A$ promoter $(-142$ to +53 ; Kumano et al. 1986). The $g \ln A$ promoter was excised from pSG2.4 (a gift from S.J. Robinson, University of Oregon, Eugenel, as a 2.4-kb HindIII-EcoRI fragment, which has its downstream end at +541 . The promoter::lux $A B$ plasmids were used to transform Synechococcus to spectinomycin resistance, transferring the reporter genes to the neutral site (Bustos and Golden 1991).

\section{Acknowledgments}

We are grateful to $T$. Suto and $H$. Kondo for their technical assistance in managing the thousands of colonies assayed in this study, and to Dr. C. Andersson for helpful comments on this paper. This research has been supported by the following grants: from the National Science Foundation (MCB-9219880 and INT9218744) to C.H.J. and (MCB-9311352) to S.S.G., from the Ministry of Education, Science and Culture, Japan (06670093 and 06807029), to T.K. and M.I., and from Japan-USA Cooperative Program (BSAR382).

The publication costs of this article were defrayed in part by payment of page charges. This article must therefore be hereby 
marked "advertisement" in accordance with 18 USC section 1734 solely to indicate this fact.

\section{References}

Allen, M.M. 1968. Simple conditions for growth of unicellular blue-green algae on plates. J. Phycol. 4: 1-4.

Bell-Pederson, D., J.C. Dunlap, and J.J. Loros. 1992. The Neurospora circadian clock-controlled gene, $c c g-2$, is allelic to eas and encodes a fungal hydrophobin required for formation of the conidial rodlet layer. Genes \& Dev. 6: 2373-2381.

Bünning, E. 1973. The physiological clock. Springer-Verlag, New York.

Bustos, S.A. and S.S. Golden. 1991. Expression of the psbDII gene in Synechococcus sp. strain PCC 7942 requires sequences downstream of the transcription start site. $J$. Bacteriol. 173: 7525-7533.

-1992. Light-regulated expression of the $p s b D$ gene family in Synechococcus sp. strain PCC 7942: Evidence for the role of duplicated $p s b D$ genes in cyanobacteria. Mol. \& Gen. Genet. 232: 221-230.

Bustos, S.A., M.R. Schaefer, and S.S. Golden. 1990. Different and rapid responses of four cyanobacterial psbA transcripts to changes in light intensity. I. Bacteriol. 172: 1998-2004.

Chlumsky, L.J. 1991. "Investigation of the structure of bacterial luciferase from Vibrio harveyi using enzymes generated by random and site-directed mutagenesis." Ph.D. thesis, Texas A\&M University, College Station, TX.

Dunlap, J.C. 1993. Genetic analysis of circadian clocks. Annu. Rev. Physiol. 55: 683-728.

Ebbole, D.J. and H. Zalkin. 1987. Cloning and characterization of a 12-gene cluster from Bacillus subtilis encoding nine enzymes for de novo purine nucleotide synthesis. I. Biol. Chem. 262: 8274-8287.

Elhai, J. and C.P. Wolk. 1988. Conjugal transfer of DNA to cyanobacteria. Methods Enzymol. 167: 747-754.

Feldman, J.F. and J.C. Dunlap. 1983. Neurospora crassa: A unique system for studying circadian rhythms. Photochem. Photobiol. Rev. 7: 319-368.

Grobbelaar, N., T.C. Huang, H.Y. Lin, and T.J. Chow. 1986. Dinitrogen-fixing endogenous rhythm in Synechococcus RF1. FEMS Microbiol, Lett. 37: 173-177.

Heintzen, C., S. Meizer, R. Fischer, S. Kappeler, K. Apel, and D. Staiger. 1994. A light- and temperature-entrained circadian clock controls expression of transcripts encoding nuclear proteins with homology to RNA-binding proteins in meristematic tissue. Plant I. 5: 799-813.

Huang, T.C. and T.-J. Chow. 1990. Characterization of the rhythmic nitrogen-fixing activity of Synechococcus sp. RF-1 at the transcription level. Curr. Microbiol. 20: 23-26.

Jacobshagen, S. and C.H. Johnson. 1994. Circadian rhythms of gene expression in Chlamydomonas reinhardtii: Circadian cycling of mRNA abundances of $c a b I I$ and possibly of $\beta$-tubulin and cytochrome c. Eur. I. Cell Biol. 64: 142-152.

Kay, S.A. and A.J. Millar. 1992. Circadian regulated $c a b$ gene transcription in higher plants. In Molecular genetics of biological rhythms (ed. M.W. Young), pp. 73-89. Marcel-Dekker, New York.

Kloppstech, K. 1985. Diurnal and circadian rhythmicity in the expression of light-induced plant nuclear messenger RNAs. Planta 165: 502-506.

Kondo, T. and M. Ishiura. 1994. Circadian rhythms of cyanobacteria: monitoring the biological clocks of individual colonies by bioluminescence. J. Bacteriol. 176: 1881-1885.

Kondo, T., C.H. Johnson, and J.W. Hastings. 1991. Action spec- trum for resetting the circadian rhythm in the CW15 strain of Chlamydomonas: I. Cells in darkness. Plant Physiol. 95: 197-205.

Kondo, T., C.A. Strayer, R.D. Kulkarni, W. Taylor, M. Ishiura, S.S. Golden, and C.H. Johnson. 1993. Circadian rhythms in prokaryotes: Luciferase as a reporter of circadian gene expression in cyanobacteria. Proc. Natl. Acad. Sci. 90: 56725676.

Kondo, T., N.F. Tsinoremas, S.S. Golden, C.H. Johnson, S. Kutsuna, and M. Ishiura. 1994. Circadian clock mutants of cyanobacteria. Science 266: 1233-1236.

Kumano, M., N. Tomioka, K. Shinozaki, and M. Sugiura. 1986. Analysis of the promoter region in the $\operatorname{rn} A$ operon from a blue-green alga, Anacystis nidulans 6301. Mol. \& Gen. Genet. 202: 173-178.

Li, R. and S.S. Golden. 1993. Enhancer activity of light-responsive regulatory elements in the untranslated leader regions of cyanobacterial psbA genes. Proc. Natl. Acad. Sci. 90: 11678-11682.

Liu, Y., S.S. Golden, T. Kondo, M. Ishiura, and C.H. Johnson. 1995. Bacterial luciferase as a reporter of circadian gene expression in cyanobacteria. J. Bacteriol. 177: 2080-2086.

Loros, J.J. and J.C. Dunlap. 1991. Neurospora crassa clock-controlled genes are regulated at the level of transcription. Mol. Cell. Biol. 11: 558-563.

Loros, J.J., S.A. Denome, and J.C. Dunlap. 1989. Molecular cloning of genes under control of the circadian clock in Neurospora. Science 243: 385-388.

Markaroff, C.A., H. Zalkin, R.L. Switzer, and S.J. Vollmer. 1983. Cloning of the Bacillus subtilis glutamine phosphoribosylpyrophosphate amidotransferase gene in Escherichia coli. J. Biol. Chem. 258: 10586-10593.

Millar, A.J. and S.A. Kay. 1991. Circadian control of $c a b$ gene transcription and mRNA accumulation in Arabidopsis. Plant Cell 3: 541-550.

Mitsui, A., S. Kumazawa, A. Takahashi, H. Ikemoto, S. Cao, and T. Arai. 1986. Strategy by which unicellular cyanobacteria grow photoautotrophically. Nature 323: 720-722.

Sambrook, J., E.F. Fritsch, T. Maniatis. 1989. Molecular cloning: A laboratory manual, 2nd ed. Cold Spring Harbor Laboratory Press, Cold Spring Harbor, New York.

Schneegurt, M.A., D.M. Sherman, S. Nayar, and L.A. Sherman. 1994. Oscillating behavior of carbohydrate granule formation and dinitrogen fixation in the cyanobacterium Cyanothece sp. strain ATCC 51142. \%. Bacteriol. 176: 15861597.

Tandeau de Marsac, N. and J. Houmard. 1993. Adaptation of cyanobacteria to environmental stimuli: New steps towards molecular mechanisms. FEMS Microbiol. Rev. 104: 119190.

Theisen, P.W., J.E. Grimwade, A.C. Leonard, J.A. Bogan, and C.E. Helmstetter. 1993. Correlation of gene transcription with the time of initiation of chromosome replication in Escherichia coli. Mol. Microbiol. 10: 575-584.

Thompson, R.J. and G. Mosig. 1990. Light affects the structure of Chlamydomonas chloroplast chromosomes. Nucleic Acids Res. 18: 2625-2631.

Tsinoremas, N.F., A.L. Kutach, C.A. Strayer, and S.S. Golden. 1994. Efficient complementation of a mutant of Synechococcus sp. strain PCC 7942 by interspecies conjugation and chromosomal recombination. J. Bacteriol. 176: 6764-6768.

Wolk, C.P., Y. Cai, and J.-M. Panoff. 1991. Use of a transposon with luciferase as a reporter to identify environmentally responsive genes in a cyanobacterium. Proc. Natl. Acad. Sci. 88: $5355-5359$. 


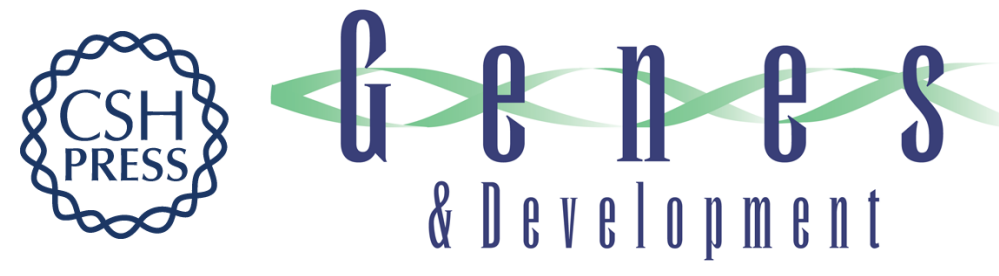

\section{Circadian orchestration of gene expression in cyanobacteria.}

Y Liu, N F Tsinoremas, C H Johnson, et al.

Genes Dev. 1995, 9:

Access the most recent version at doi:10.1101/gad.9.12.1469

References This article cites 32 articles, 17 of which can be accessed free at:

http://genesdev.cshlp.org/content/9/12/1469.full.html\#ref-list-1

License

Email Alerting Receive free email alerts when new articles cite this article - sign up in the box at the top Service right corner of the article or click here.

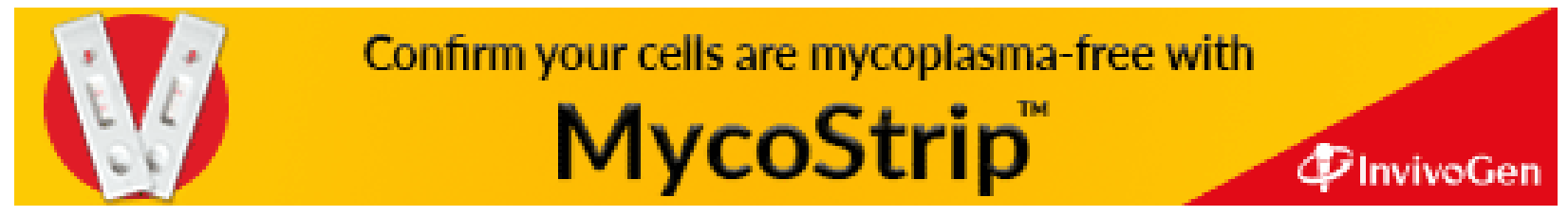

